\title{
MUDANÇAS POSICIONAIS DA LINHA DE COSTA NAS PRAIAS DO PINA E DE BOA VIAGEM, RECIFE, PE, BRASIL
}

\author{
Maria das Neves GREGÓRIO1*; Tereza Cristina Medeiros de ARAÚJO²; Francisco Jaime Bezerra \\ MENDONÇA3; Rodrigo MIKOSZ GONÇALVES ${ }^{3}$; Rafael Lopes MENDONÇA ${ }^{3}$
}

\begin{abstract}
${ }^{1}$ Instituto de Tecnologia de Pernambuco. Av. Prof. Luís Freire, 700, CEP 50.740-540, Recife, PE, Brasil. Email: neves.gregorio@itep.br.

*Autor correspondente

${ }^{2}$ Departamento de Oceanografia, Universidade Federal de Pernambuco. Av. Arquitetura, s/n, CEP 50.740550, Recife, PE, Brasil. E-mail: terezaraujo.ufpe@gmail.com.

${ }^{3}$ Departamento de Engenharia Cartográfica, Universidade Federal de Pernambuco. Av. Acadêmico Hélio Ramos, s/n, CEP 50.740-530, Recife, PE, Brasil. E-mail: jaime@ufpe.br, rodrigo.mikosz@ufpe.br, rafaellomendonca@gmail.com.

RESUMO. O objetivo deste trabalho foi analisar a evolução espacial e temporal da linha de costa considerando um intervalo de 48 anos $(1960-2008)$ para as praias urbanas do Pina e de Boa Viagem (Recife, PE, Brasil). O levantamento multitemporal da linha de costa foi baseado na análise de dados vetoriais fotogramétricos (1960,1974,1981, 1997 na escala 1: 6.000) e dados coletados por GNSS para 0 ano de 2008. A área de estudo foi dividida em quatro setores (A, B, C e D). Como resultados o setor A obteve taxas de retrogradação onde o valor para a maior taxa foi de $-1,8 \mathrm{~m} /$ ano (1960-1974). Para o setor B destaca-se a progradação para o intervalo de 1960-1974 (2,5 m/ano). As maiores taxas de retrogradação se encontram no setor C $(-0,87 ;-0,56 ;-0,44$, durante os períodos de 1960-1981, 19601997 e 1960-2008 respectivamente). O setor D obteve como resultado a retrogradação para todos os intervalos analisados, sendo que a maior taxa encontrada foi para o de 1960-1974 (-1,6 m/ano). Por fim, observou-se que não há uma relação linear em relação às mudanças da linha de costa para os setores analisados demonstrando o complexo comportamento hidro-sedimentar destas praias urbanas.
\end{abstract}

Palavras-Chave: monitoramento costeiro, análise multi-temporal, erosão marinha.

\begin{abstract}
The objective of this study was to analyze the spatial and temporal evolution of the shoreline considering an interval of 48 years (1960-2008) for the urban beaches of Pina and Boa Viagem (Recife, PE, Brazil). The multi-temporal shoreline survey was supported by vector analysis using photogrammetric data (1960, 1974, 1981, 1997, scale 1: 6,000) and GNSS observations collected for the year 2008. The study area was divided in four sectors $(A, B, C$ and $D)$. As results, the sector $A$ showed retrogradation rates where the value for the highest rate was $-1.8 \mathrm{~m} /$ year (1960-1974). For sector $B$ highlight progradation for the interval 1960-1974 (2.5 m/years). The larger retrogradation rates found in sector C $(-0.87 ;-0.56 ;-0.44$, during the periods 1960-1981, 1960-1997 and 1960-2008, respectively). The sector D had as results retrogradation for all periods analyzed, where the highest rate was found for the period 1960-1974 (-1.6 $\mathrm{m} /$ year). Finally, it was possible to observe that there is not a linear relationship regarding shoreline changes for the periods and sectors analyzed demonstrating the complex hydro-sedimentary behavior of these urban beaches.
\end{abstract}

Keywords: coastal monitoring, multi-temporal analysis, marine erosion. 


\section{INTRODUÇÃO}

Muitas praias são conhecidas por possuírem ocorrência de erosão costeira. Estima-se que $70 \%$ das zonas costeiras com praias arenosas do mundo estão sofrendo processos erosivos, $10 \%$ estão em progradação e 20 \% encontram-se em relativa estabilidade (Bird, 1996). Os processos de erosão e deposição dependem de vários fatores, tais como: a taxa de suprimento de sedimentos e a declividade da zona costeira, podendo levar à acumulação de grandes corpos clásticos arenosos, desenvolvidos abaixo e acima do nível da água, sendo denominados de áreas litorâneas (Villwock et al., 2005).

A dinâmica sedimentar atual responde pelas variações da linha de costa. Esta dinâmica é regida principalmente por condicionantes oceanográficas astronômicas/meteorológicas: o clima de ondas, o regime de marés e a deriva litorânea. Sendo esta última o processo mais relativo de transporte de sedimentos ao longo das costas arenosas e qualquer modificação introduzida pelo homem afeta o equilíbrio de estoque natural de sedimentos das praias, alterando as taxas de erosão e deposição. São exemplos das alterações antrópicas que afetam a dinâmica sedimentar a construção de molhes, portos, aterros e drenagens (Villwock et al., 2005).

Estudos sobre alterações da configuração espacial da linha de costa têm sido realizados em várias partes do mundo, como, por exemplo, no Sul do Mar Báltico (Schwarzer and Schrottke, 1999), na Flórida (Miller and Dean, 2004) e em Portugal (Sousa, 2004; Oliveira, 2005; Ferreira et al., 2006), entre outros.

Há vários exemplos de estudos regionais no Brasil que retratam pesquisas considerando taxas de variações na posição espacial da linha de costa, características morfodinâmicas locais e fazendo uso de informações geográficas. Como no estado do Rio Grande do Sul (Baitelli et al., 1999; Esteves et al., 2002; Toldo Jr. et al., 2005), em Santa Catarina (Mazzer and Dillemburg, 2009), no Rio Grande do Norte (Lima et al., 2001; Grigio, 2003; Lima, 2004; Chaves, 2005), no Ceará (Bezerra et al., 2003), no Paraná (Krueger et al., 2009; Gonçalves et al., 2010; Tanajura et al., 2011; Krueger et al., 2011; Gonçalves et al., 2012a,b) e em Pernambuco (Muehe, 1998; Duarte, 2002; Gregório, 2004, Gregório et al., 2004; Mendonça, 2005; Pedrosa, 2007; Gregório and Araújo, 2008; Santos, 2008; Gonçalves et al., 2013; Silva et al., 2013; Mendonça et al., 2014), entre outros.

As praias em Recife, Pernambuco, foram locais da exploração turística a partir da década de 70 acelerando o processo de urbanização. Sem planejamento adequado, a orla de Boa Viagem e da praia do Pina passaram a ser exploradas não só pelas atividades turísticas, mas também por atividades imobiliárias, resultando na perda das características ambientais (Silva et al., 2006).

O objetivo deste estudo foi determinar a evolução multitemporal, ao longo de 48 anos (1960-2008), através da taxa de variação da linha de costa obtendo assim sua progradação e retrogradação nas praias do Pina e de Boa Viagem. Um dos fatores que motivou o estudo foi a necessidade do monitoramento da posição da linha de costa de tais praias, devido ao processo erosivo atuante. Além disso, são praias com a urbanização consolidada de grande valor econômico 
para a região tendo um papel importante para a sociedade e para o turismo que aproveita sua beleza cênica para atrair visitantes.

\section{MATERIAL E MÉTODOS \\ ÁREA DE ESTUDO}

O estado de Pernambuco possui uma faixa costeira de $187 \mathrm{~km}$ de extensão, com um formato alongado, na direção N-S. Este litoral está inserido no contexto geomorfológico no macro compartimento denominado Costa dos Tabuleiros Norte, que compreende parte das bacias sedimentares da Paraíba, de Pernambuco e do Cabo (Barbosa et al., 2003) sendo estas separadas pelo Lineamento Pernambuco, que corta a cidade do Recife. Estas bacias são preenchidas por sedimentação meso-cenozóica, representadas pelas formações Cabo, Beberibe e Gramame e pela Formação Barreiras.

Nos bairros de Boa Viagem, Pina e Brasília Teimosa ocorrem terraços marinhos holocênicos, limitados pelas áreas de manguezais, pelo canal do Setúbal e pela linha de costa, constituídos por areias quartzosas claras. Próximo a linha de costa, são encontradas linhas de recifes de arenitos paralelos a faixa de praia.

Os bancos de arenitos, rocha praial ou beachrocks (Fig. 1) são feições lineares que se estendem, às vezes, continuamente, por cerca de $10 \mathrm{~km}$, com uma largura média variando de 20 a $60 \mathrm{~m}$, e 3 a $4 \mathrm{~m}$ de espessura. Afloram na baixa-mar ou mesmo acima do nível médio do mar, e se dispõem paralelamente à linha de costa (Dominguez et al., 1990). Segundo Mabesoone (1964), os bancos de arenitos são constituídos de $20 \%$ a $80 \%$ de areias de quartzo, e o restante constituído de fragmentos carbonáticos, principalmente de moluscos e algas, a parte submarina da praia, muito rica em calcário, que pelas diversas etapas de regressão marinha se consolida na vizinhança do lençol freático com temperaturas suficientemente altas para permitir a precipitação do carbonato de cálcio como cimento. Segundo Delibrias and Laborel (1971), os recifes têm uma idade de 5.900 mais ou menos 300 A.P. e podem atuar protegendo a costa do efeito da energia das ondas, gerando processos erosivos em suas extremidades ou podendo gerar processos de refração, que acabam por aumentar a energia das ondas. 

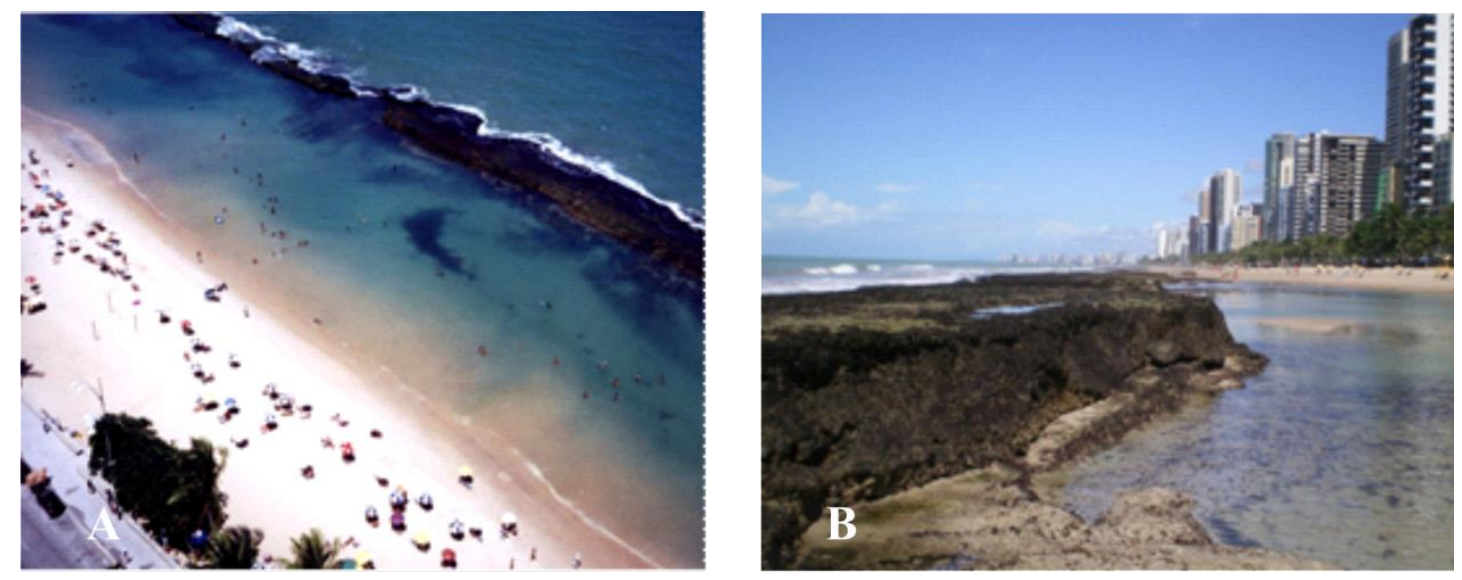

Figura 1. Vista área dos bancos de arenitos da praia de Boa Viagem (A). Os bancos de arenitos localizados na praia do Pina $(B)$.

A cidade do Recife possui um clima, segundo a classificação de Köppen, quente e úmido (As'), com temperatura média de $26^{\circ} \mathrm{C}$ e pluviosidade média anual de $1.720 \mathrm{~mm}$ (IBGE, 1997). O ritmo sazonal da costa do Nordeste brasileiro é definido por dois regimes distintos sendo uma fase de chuvas com pluviosidade superior a $100 \mathrm{~mm}$, e outro seco, com precipitações inferiores a $100 \mathrm{~mm}$. Os ventos indicam uma predominância na direção sudeste com maior variância entre SE/E durante a época seca e SE/S durante a chuvosa. Os ventos são um dos principais formadores das ondas e estas influenciam na dinâmica do ambiente praial.

O clima de ondas pode ser deduzido pelo conhecimento dos mecanismos responsáveis que geram as correntes no Atlântico Sul e áreas de tempestades nas altas latitudes. A costa lestenordeste do Brasil é controlada pelo anticiclone estacionário Atlântico Sul. Os ventos que alcançam a costa leste e nordeste vêm de duas maiores direções: a) E-SE; e b) NE. No setor compreendido entre Cabo Frio ( $R J)$ e a costa de Pernambuco, as ondas mais efetivas no transporte sedimentar são geradas pelos ventos alísios, do quadrante nordeste e leste, com período médio de 5 a 10 segundos e altura entre 1 e 2 metros (Tessler and Goya, 2005).

Pequenas mudanças no regime de ondas afetam o ângulo de incidência e a direção do transporte ao longo da costa, e tem importantes implicações no estoque sedimentar ao longo da zona costeira, ativando ou acelerando processos erosivos (Martin et al., 1998).

Neste trabalho, a área em estudo compreende as praias do Pina e de Boa Viagem, localizadas no ambiente litorâneo da cidade do Recife, PE, no Setor Médio da costa pernambucana, com um formato alongado, na direção N-S. As praias do Pina e de Boa Viagem compreendem uma área de 57,48 hectares, com uma extensão de 8 km, entre as coordenadas planas 9106000 - $9097000 \mathrm{~N}$ e 294000 - 287000 E (Fig. 2). 

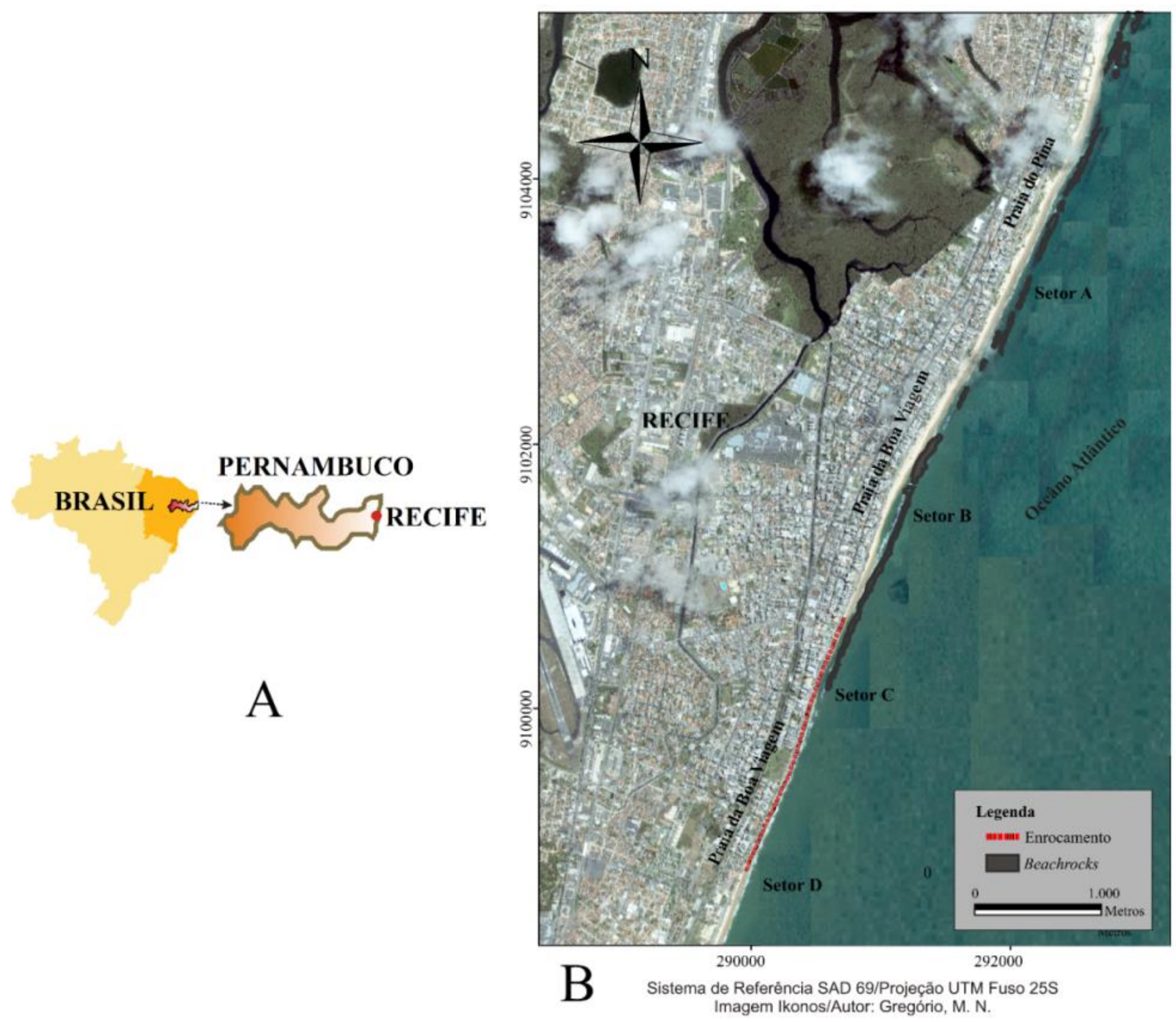

Figura 2. Localização do estado de Pernambuco e sua capital no Brasil (A). Localização das praias urbanas do Recife: Boa Viagem e Pina (B).

O litoral da cidade do Recife vem sofrendo processos de erosão marinha, principalmente na região sul (Praia de Boa Viagem) que vem sendo urbanizada nas últimas décadas. Ao norte da praia de Boa Viagem, a praia do Pina, tem um ambiente praial constituído por regiões de dunas frontais, pós-praia e face da praia consideradas como preservadas (Fig. 3). Entretanto, este ambiente estreita-se em direção ao Sul, desaparecendo as dunas frontais e demarcando o início de um processo erosivo com obras de contenção do tipo enrocamento (Fig. 4). Atualmente conta com uma obra de contenção, iniciada em 1995 e prolongada para o Norte durante os anos de 2004 e 2008 (Fig. 5). A Prefeitura do Recife tem despesas constantes para manutenção e remobilização dos blocos de granitos (enrocamento). 

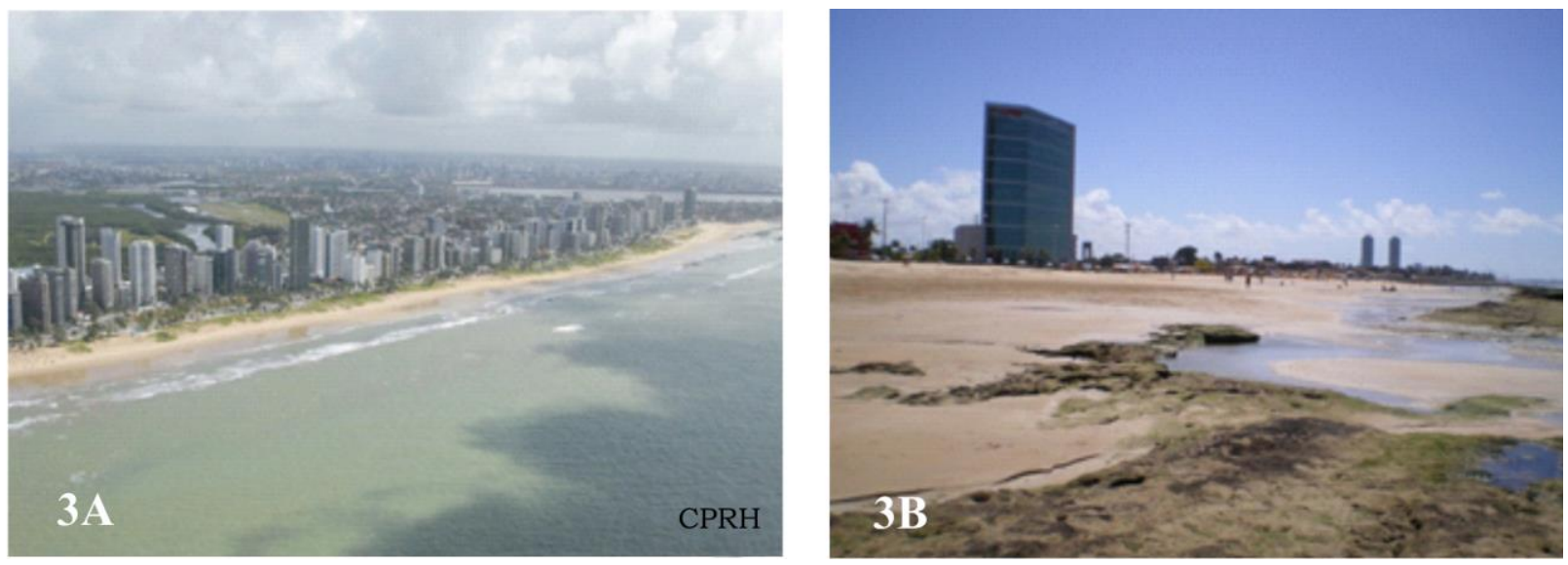

Figura 3. Vista área das praias do Pina e de Boa Viagem (A). Vista em direção norte da praia do Pina (B).
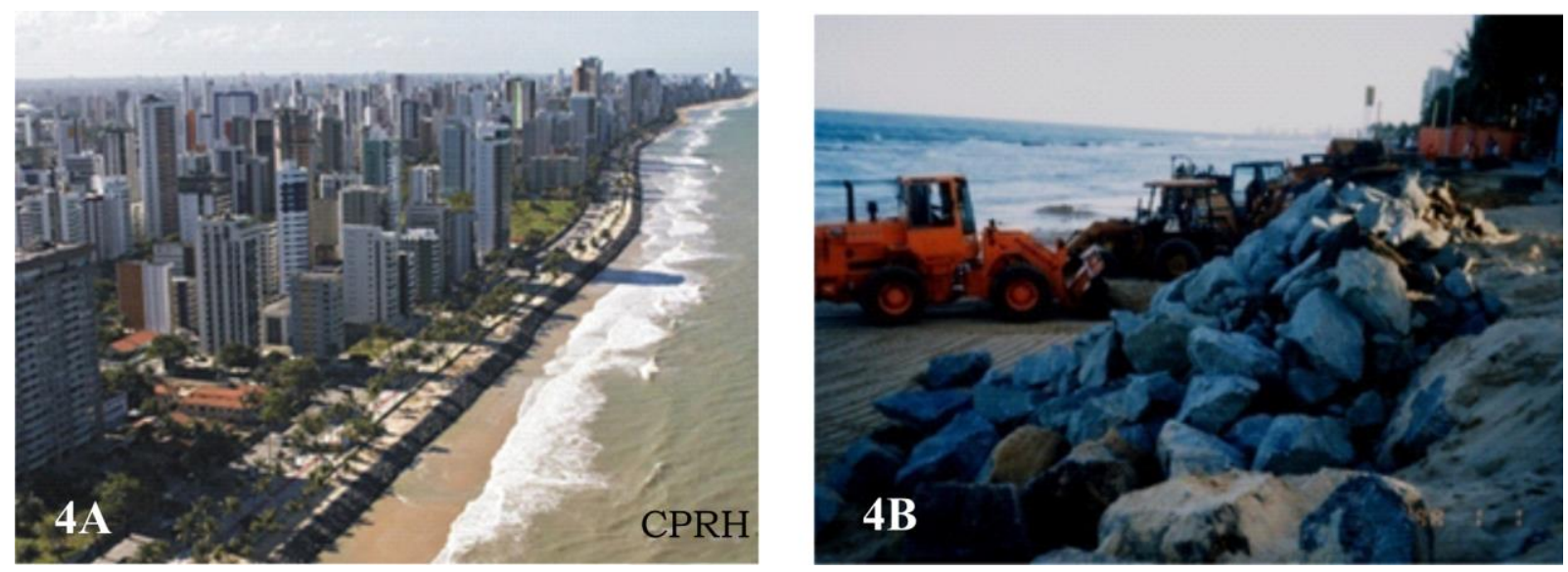

Figura 4. Vista área da obra de contenção da praia de Boa Viagem (A). Manutenção do enroncamento em Boa Viagem (B).
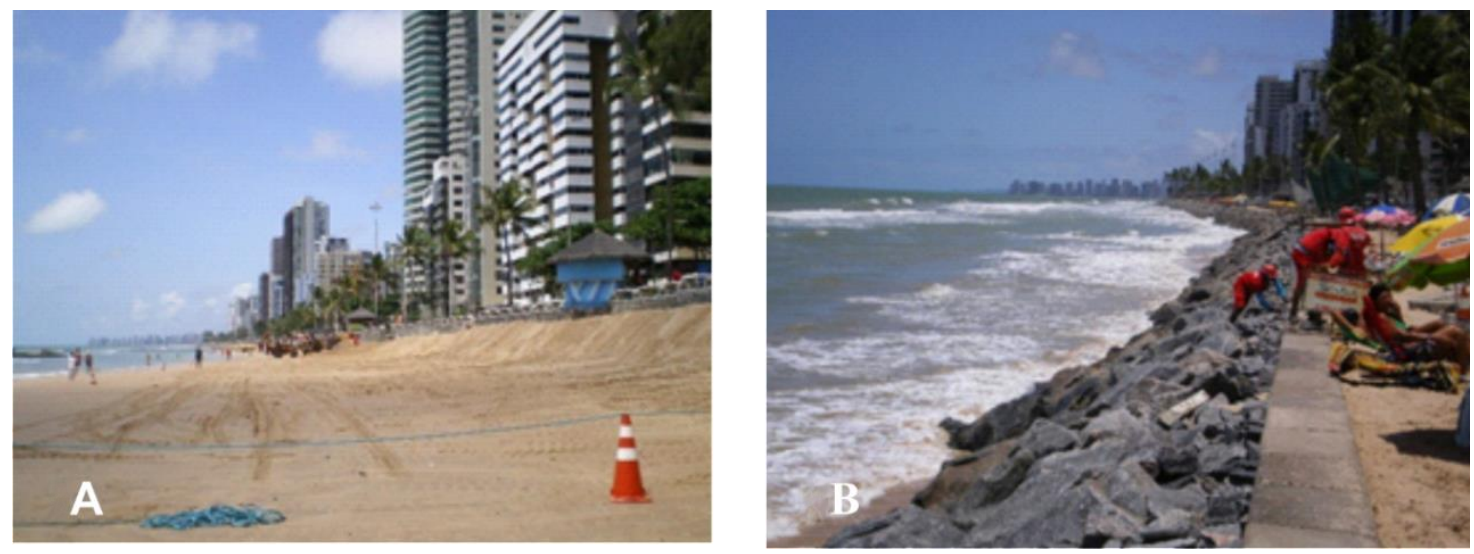

Figura 5. Indicativos do processo erosivo em 2008 (A). Manutenção da obra de contenção na praia de Boa Viagem (B).

Estudos de correntometria realizados nos largos das praias de Boa Viagem e Piedade, utilizando quatro estágios de maré demonstram que o transporte líquido à superfície é para o sentido Sul. O transporte maior se dá próximo a costa. Na camada intermediária encontra-se um 
transporte costa-a-fora em toda área de 5,8 a 31,6 cm. $\mathrm{s}^{-1}$. O transporte de fundo é menos intenso, variando de 7,2 a 21,9 cm.s ${ }^{-1}$ e é em direção à costa, exceto próximo a foz do rio Jaboatão, que se direciona para o mar (Rollnic, 2002). Na região de Boa Viagem há predominância das correntes, na direção de Sul para Norte, e contra a costa, nas camadas ao fundo e superficial, durante os meses com chuva. Já na estiagem, há predominância na direção de Norte para Sul e costa-a-fora, nas camadas de fundo e superficial (Rollnic, 2002).

\section{INFORMAÇÕES TEMPORAIS}

Para determinar as variações da linha de costa utilizaram-se informações de fotografias aéreas métricas para os anos de 1960, 1974, 1981, 1997 e a linha de costa extraída por GNSS (Global Navigation Satellite Systems) de 2008, totalizando desta forma um estudo com um intervalo de 48 anos. As fotografias aéreas do ano de 1960 foram adquiridas pelo Departamento de Oceanografia da Universidade Federal de Pernambuco (UFPE), em formato impresso, na escala 1:6.000, sendo posteriormente processadas para o formato digital. Para os anos de 1974, 1981, 1997, os autores conseguiram este material junto a Fundação de Desenvolvimento da Região Metropolitana do Recife (FIDEM), em formato digital, na escala 1:6.000. A linha de costa para o ano de 2008 foi mapeada através do levantamento geodésico GNSS no modo relativo cinemático pelo Departamento de Engenharia Cartográfica da UFPE e cedidas para o projeto MAI, (Monitoramento Ambiental Integrado), também realizado pela UFPE.

Todas as imagens foram devidamente processadas e georreferenciadas no mesmo sistema geodésico de referência. Mais detalhes sobre o processo de registro de imagens podem ser encontrados em Ribeiro et al. (2004). Neste trabalho utilizou-se como indicativo da linha de costa, o alcance máximo da maré na tomada das fotografias aéreas identificado por especialistas através da linha de deixa. Para cada ano, 1974, 1981, 1997 e 2008 (Fig. 6), foram extraídas as respectivas posições da linha de costa e posteriormente comparadas com a linha de base considerando a do ano de 1960.

Considerou-se que o erro computado no processo de georreferenciamento e extração da linha de costa nas fotografias aéreas foi em média de 2,50 m, valor este estimado em função da escala da foto e do erro do operador. Destaca-se que mesmo contendo erros posicionais tanto no processo de registro das imagens e na extração da linha de costa temporal a fonte de informações cartográficas torna-se fundamental para os estudos costeiros e dependendo da análise geomorfológica proposta, pode ser utilizada com uma precisão métrica (Gonçalves, 2010; Gonçalves et al., 2013). Por outro lado, levantamentos geodésicos realizados com o emprego do GNSS podem alcançar precisão centimétrica.

\section{CÁlCULO dA VARIAÇÃo dA LINHA DE COSTA}

O cálculo das taxas de variação da linha de costa (m/ano) considerando os sentidos de progradação e retrogradação, foram realizados no modo automático na extensão DSAS (Digital 
Shoreline Analysis System) Thieler et al. (2009), através das distâncias perpendiculares entre a linha de costa considerada como base (1960) e as demais linhas de costa (1974, 1981, 1997, 2008). As distâncias consideradas para cada transecto foram de 5 em $5 \mathrm{~m}$. A partir dos resultados encontrados foram calculadas as estatísticas descritivas como: a média aritmética, a mediana, a variância, o desvio padrão e a correlação linear de Pearson entre os setores analisados.

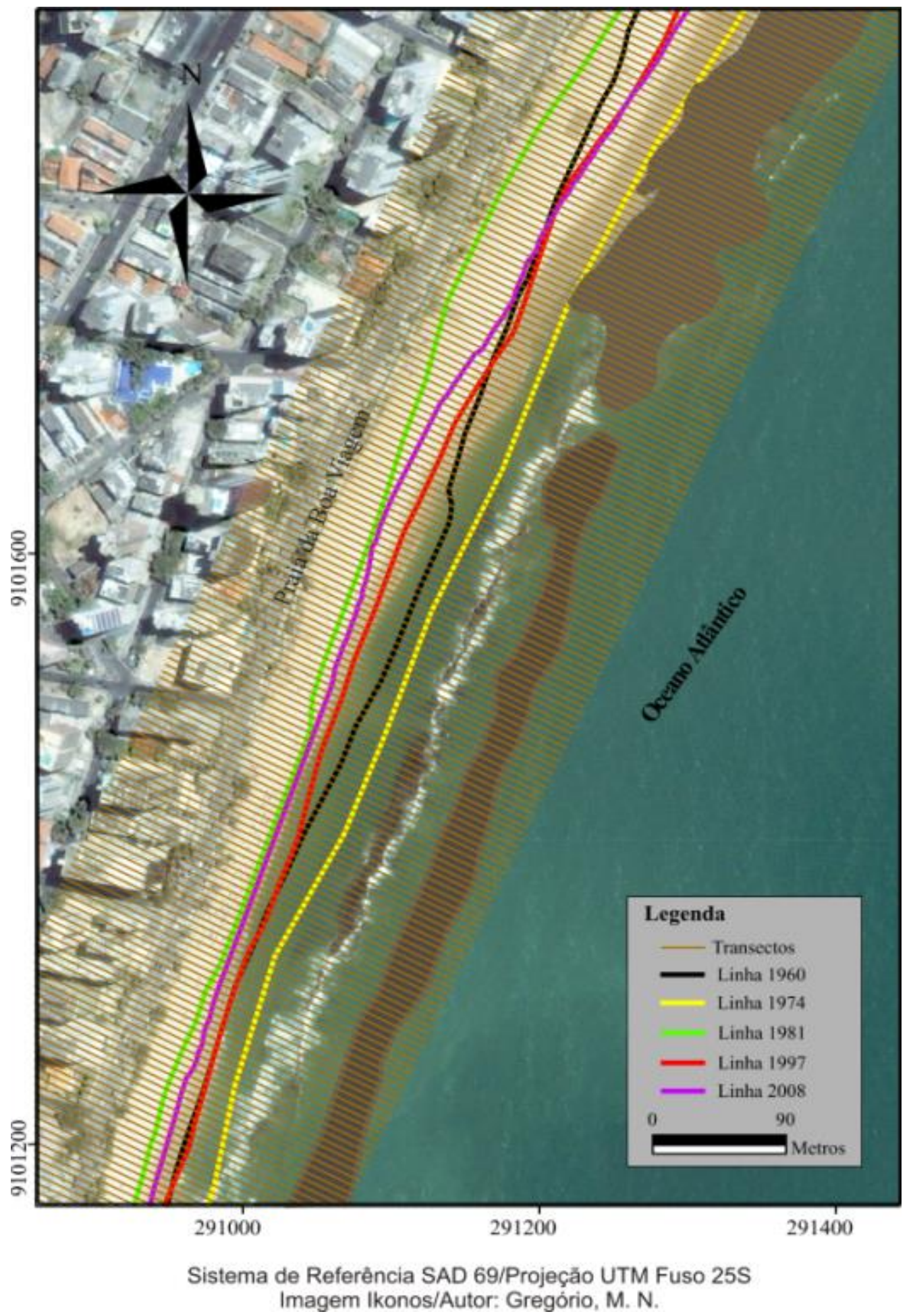

Figura 6. Linhas de costa temporais e representação dos transectos. 
A área de estudo foi dividida em quatro setores. O setor A, compreende a praia do Pina, utilizando como início da divisão com a parte norte da praia de Boa Viagem à Rua Henrique Capitulino; o setor B, da Rua Henrique Capitulino à Casa da Aeronáutica, número 4224 da Av. Boa Viagem; o setor C, da Casa da Aeronáutica ao norte do Parque Dona Lindu e o setor D ao norte do Parque Dona Lindu ao limite do município do Jaboatão dos Guararapes (Fig. 7).

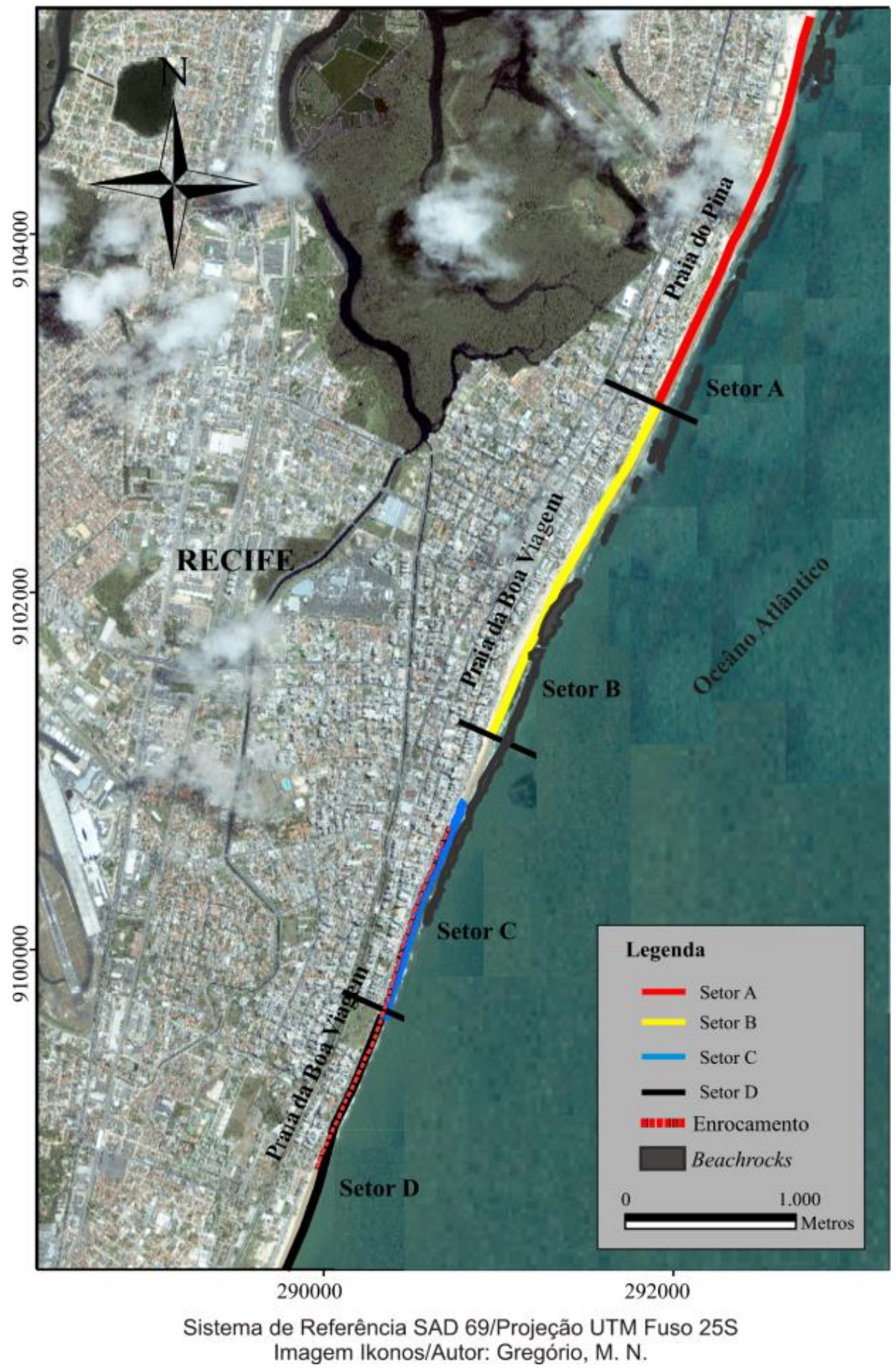

Figura 7. Localização dos setores A, B, C e D. 


\section{RESULTADOS}

Os resultados dos deslocamentos médios, medianos, máximo, mínimo, variância e desvio padrão entre as linhas de costa considerado o respectivo setor estão presentes na tabela 1 . Os maiores valores absolutos encontrados para os deslocamentos entre as linhas de costa foram observados no setor A, durante 1960-1974 com 73,36 m e 58,80 m entre 1960-1981. Os valores obtidos na tabela 1 foram utilizados para calcular as taxas de variação da linha de costa na tabela 2 , dividindo o valor do deslocamento encontrado pelo intervalo de tempo.

Tabela 1. Estatísticas do deslocamento da linha de costa.

\begin{tabular}{|c|c|c|c|c|c|c|c|}
\hline \multicolumn{2}{|c|}{ Setores/Períodos } & $\begin{array}{c}\text { Média } \\
(\mathrm{m})\end{array}$ & $\begin{array}{l}\text { Mediana } \\
(\mathrm{m})\end{array}$ & $\begin{array}{l}\text { Mínimo } \\
(\mathrm{m})\end{array}$ & $\begin{array}{l}\text { Máximo } \\
(\mathrm{m})\end{array}$ & $\begin{array}{c}\text { Variância } \\
\left(\mathrm{m}^{2}\right)\end{array}$ & $\begin{array}{c}\text { Desvio } \\
\text { Padrão }(\mathrm{m})\end{array}$ \\
\hline \multirow{4}{*}{$\begin{array}{l}\varangle \\
\vdots \\
\text { ¿ } \\
\text { ஸे }\end{array}$} & $1960-1974$ & $-25,73$ & $-27,16$ & $-73,36$ & 21,14 & 590,93 & 24,31 \\
\hline & $1960-1981$ & $-15,98$ & $-17,01$ & $-58,80$ & 20,37 & 460,60 & 21,46 \\
\hline & $1960-1997$ & $-2,77$ & $-4,07$ & $-37,00$ & 42,55 & 351,58 & 18,75 \\
\hline & $1960-2008$ & $-12,56$ & $-13,44$ & $-51,36$ & 43,20 & 445,03 & 21,10 \\
\hline \multirow{4}{*}{ 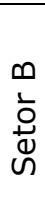 } & $1960-1974$ & 34,99 & 36,26 & 15,40 & 49,42 & 97,27 & 9,86 \\
\hline & $1960-1981$ & $-13,11$ & $-14,91$ & $-42,42$ & 10,29 & 174,46 & 13,21 \\
\hline & $1960-1997$ & 1,08 & 1,48 & $-14,06$ & 15,54 & 41,16 & 6,42 \\
\hline & $1960-2008$ & $-1,78$ & $-3,36$ & $-14,40$ & 17,76 & 73,99 & 8,60 \\
\hline \multirow{4}{*}{ 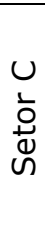 } & $1960-1974$ & 5,88 & 2,66 & $-11,06$ & 32,20 & 146,29 & 12,09 \\
\hline & $1960-1981$ & $-18,30$ & $-19,95$ & $-34,02$ & $-4,41$ & 70,93 & 8,42 \\
\hline & $1960-1997$ & $-20,57$ & $-23,68$ & $-35,15$ & 2,22 & 71,32 & 8,45 \\
\hline & $1960-2008$ & $-21,07$ & $-21,12$ & $-36,48$ & $-6,24$ & 54,33 & 7,37 \\
\hline \multirow{4}{*}{ 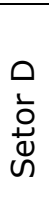 } & $1960-1974$ & $-22,48$ & $-22,26$ & $-34,02$ & $-7,84$ & 51,47 & 7,17 \\
\hline & $1960-1981$ & $-24,50$ & $-23,10$ & $-40,74$ & $-8,61$ & 88,20 & 9,39 \\
\hline & $1960-1997$ & $-6,96$ & $-0,74$ & $-41,44$ & 11,10 & 255,33 & 15,98 \\
\hline & $1960-2008$ & $-9,35$ & $-7,2$ & $-29,28$ & 4,32 & 104,5 & 10,22 \\
\hline
\end{tabular}

Na tabela 2 são demonstrados para cada setor os resultados para médias das taxas de variação da linha de costa, a mediana, os valores máximos e mínimos, a variância e o desvio padrão. O setor A obteve o maior valor médio nas taxas de retrogradação da linha de costa com -1,84 m/ano entre 1960-1974, para as demais épocas 1960-1981, 1960-1997, 1960-2008 também foram encontradas taxas de variações negativas conforme os valores na tabela 2 . 0 valor mínimo encontrado considerando todos os setores foi de -5,24 m/ano (1960-1974) pertencente ao setor A e o máximo de 3,53 m/ano para o setor B entre 1960-1974.

O setor B apresentou valor médio positivo (progradação) na taxa de variação da linha de costa entre 1960-1974 com 2,50 m/ano, bem como a maior distância de avanço considerando o intervalo de 1960-1974 com 49,42 m. A partir de 1960-1981 obteve-se uma retrogradação com uma taxa de $-0,62 \mathrm{~m} / \mathrm{ano}$, posteriormente as taxas foram consideradas estáveis com 0,03 m/ano e -0,04 m/ano entre 1960-1997 e 1960-2008, respectivamente. 
As taxas de variação da linha de costa consideradas como retrogradação no setor C foram obtidas considerando 1960-1981, 1960-1997 e 1960-2008, onde o maior valor desta taxa ocorreu entre 1960-1981 com -0,87 m/ano. Durante o período de 1960-1974 detectou-se um avanço com uma taxa de 0,42 m/ano.

Tabela 2. Taxas de variação da linha de costa e estatísticas para os setores.

\begin{tabular}{|c|c|c|c|c|c|c|c|}
\hline \multicolumn{2}{|c|}{ Setores/Períodos } & $\begin{array}{c}\text { Taxas Média } \\
\text { (m/ano) }\end{array}$ & $\begin{array}{l}\text { Mediana } \\
\text { (m/ano) }\end{array}$ & $\begin{array}{c}\text { Mínimo } \\
\text { (m/ano) }\end{array}$ & $\begin{array}{l}\text { Máximo } \\
\text { (m/ano) }\end{array}$ & $\begin{array}{c}\text { Variância } \\
\left(\mathrm{m}^{2}\right)\end{array}$ & $\begin{array}{c}\text { Desvio } \\
\text { Padrão }(\mathrm{m})\end{array}$ \\
\hline \multirow{4}{*}{ 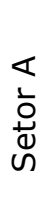 } & $1960-1974$ & $-1,84$ & $-1,94$ & $-5,24$ & 1,51 & 3,01 & 1,74 \\
\hline & $1960-1981$ & $-0,76$ & $-0,81$ & $-2,80$ & 0,97 & 1,04 & 1,02 \\
\hline & $1960-1997$ & $-0,07$ & $-0,11$ & $-1,00$ & 1,15 & 0,26 & 0,51 \\
\hline & $1960-2008$ & $-0,26$ & $-0,28$ & $-1,07$ & 0,90 & 0,19 & 0,44 \\
\hline \multirow{4}{*}{ 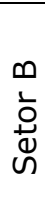 } & $1960-1974$ & 2,50 & 2,59 & 1,10 & 3,53 & 0,50 & 0,70 \\
\hline & $1960-1981$ & $-0,62$ & $-0,71$ & $-2,02$ & 0,49 & 0,40 & 0,63 \\
\hline & $1960-1997$ & 0,03 & 0,04 & $-0,38$ & 0,42 & 0,03 & 0,17 \\
\hline & $1960-2008$ & $-0,04$ & $-0,07$ & $-0,30$ & 0,37 & 0,03 & 0,18 \\
\hline \multirow{4}{*}{ 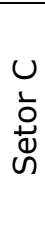 } & $1960-1974$ & 0,42 & 0,19 & $-0,79$ & 2,30 & 0,75 & 0,86 \\
\hline & $1960-1981$ & $-0,87$ & $-0,95$ & $-1,62$ & $-0,21$ & 0,16 & 0,40 \\
\hline & $1960-1997$ & $-0,56$ & $-0,64$ & $-0,95$ & 0,06 & 0,05 & 0,23 \\
\hline & $1960-2008$ & $-0,44$ & $-0,44$ & $-0,76$ & $-0,13$ & 0,02 & 0,15 \\
\hline \multirow{4}{*}{ 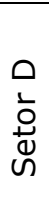 } & $1960-1974$ & $-1,61$ & $-1,59$ & $-2,43$ & $-0,56$ & 0,26 & 0,51 \\
\hline & $1960-1981$ & $-1,13$ & $-1,06$ & $-1,94$ & $-0,41$ & 0,17 & 0,41 \\
\hline & $1960-1997$ & $-0,19$ & $-0,02$ & $-1,12$ & 0,30 & 0,19 & 0,43 \\
\hline & $1960-2008$ & $-0,19$ & $-0,15$ & $-0,61$ & 0,09 & 0,05 & 0,21 \\
\hline
\end{tabular}

Foi possível estimar o valor da área considerada como retrogradação ou progradação. No caso do recuo da linha de costa por ano (Tab. 3), foi constatado que os maiores valores por área foram encontrados para 1960-1974 e 1960-1981, evidenciado na soma de todos os setores, correspondendo a 135,76 $\mathrm{m}^{2}$ e 147,94 $\mathrm{m}^{2}$ respectivamente. Nos setores A e C destacam-se os maiores valores de 208,701 m² e 102,109 m², respectivamente. Nos setores C e D evidenciaramse os menores índices de progradação para 1960-1974 e 1960-1997. Destaca-se que no setor C, a linha de costa teve um comportamento de retrogradação em todos os intervalos considerados.

O setor D obteve médias negativas nas taxas de variação da linha de costa, para todas épocas, sendo as maiores encontradas para os anos de 1960-1974 com -22,48 m e 1960-1981 com -24,50 m. Os menores valores encontram-se entre 1960-1997 e 1960-2008, ambos com o mesmo valor de $-0,19 \mathrm{~m} /$ ano. Para os deslocamentos entre linhas de costa neste setor verificouse o maior valor para a progradação durante 1960-1997 com 11,10 m, diminuindo entre 19602008 para $4,32 \mathrm{~m}$.

Em relação ao desvio padrão das médias os maiores valores encontrados foram para os setores A e C durante 1960-1974, com 1,74 m e 0,86 m, respectivamente. O setor A, em todas 
as épocas obteve os maiores valores de desvio padrão nas distâncias dos transectos. O maior valor obtido para o desvio padrão foi de 24,31 m entre os anos de 1960-1974.

Os valores entre a média e a mediana nas distâncias dos transectos ficaram próximos no setor A, com média de -25,73 e mediana -27,16m (1960 a 1974). Entretanto, constata-se neste mesmo setor um alto grau de dispersão, com o desvio padrão alto $(24,31 \mathrm{~m})$. Considerando a variância e ao grau de dispersão, os demais intervalos e setores tiveram valores menores do que o setor $A$.

Tabela 3. Áreas de acreção e erosão da linha de costa conforme o período.

\begin{tabular}{lcccccccccc}
\hline $\begin{array}{l}\text { Setores/ } \\
\text { Período }\end{array}$ & $\begin{array}{c}\text { Setor } \\
\text { A }\end{array}$ & $\begin{array}{c}\text { Setorão/m } \\
\text { B }\end{array}$ & $\begin{array}{c}\text { Setor } \\
\text { C }\end{array}$ & $\begin{array}{c}\text { Setor } \\
\text { D }\end{array}$ & $\begin{array}{c}\text { Setor } \\
\text { A }\end{array}$ & $\begin{array}{c}\text { Setor } \\
\text { B }\end{array}$ & $\begin{array}{c}\text { Setor } \\
\text { C }\end{array}$ & $\begin{array}{c}\text { Setor } \\
\text { D }\end{array}$ & $\begin{array}{c}\text { Total/ } \\
\text { Erosão } \\
\left(\mathrm{m}^{2}\right)\end{array}$ & $\begin{array}{c}\text { Total/ } \\
\text { Progradação } \\
\left(\mathrm{m}^{2}\right)\end{array}$ \\
\hline $1960-$ & 94,616 & 0 & 3,425 & 37,723 & 13,023 & 72,66 & 13,16 & 0,00 & 135,76 & 98,85 \\
1974 & & & & & & & & & & \\
$1960-$ & 48,433 & 29,67 & 30,15 & 39,687 & 7,266 & 2,333 & 0,00 & 0,00 & 147,94 & 9,59 \\
1981 & & & & & & & & & & \\
$1960-$ & 23,651 & 4,127 & 33,85 & 19,624 & 17,328 & 6,413 & 0,56 & 5 & 81,25 & 29,30 \\
1997 & & & & & & & & & & \\
$1960-$ & 42,002 & 9,555 & 34,68 & 18,621 & 9,711 & 5,856 & 0,00 & 0,03 & 104,85 & 15,60 \\
2008 & & & & & & & & & & \\
& & & & & & & & & & $\mathrm{~m}^{2} / 48$ anos \\
TOTAL & 208,7 & 43,35 & 102,1 & 115,6 & 47,327 & 87,27 & 13,73 & 5,039 & 469,82 & 153,362 \\
\hline
\end{tabular}

Os resultados do coeficiente de correlação de Pearson entre os setores (Tab. 4) indicam em uma primeira análise que não houve uma correlação linear de progradação ou retrogradação nas taxas de variação da linha de costa. Entretanto, considerando setores separados foi possível identificar uma relação linear negativa para B e A com -0,66 (1960-1974), C e B com -0,85 (19601974) e D e C com -0,79 (1960-1997). Por fim, apenas duas relações lineares positivas entre D e C, com 0,62 (1960-1981) e 0,67 (1960-2008).

\section{DISCUSSÃo}

Os sedimentos que se encontram junto a linha de costa, estão em constante movimentação, procurando sua posição no perfil de equilíbrio em função das características geomorfológicas e hidrodinâmicas do ambiente costeiro. A troca de estoques de areia entre a porção emersa e submersa do perfil praial, ocorre principalmente durante os ventos de tempestade que normalmente são mais significativos em regiões temperadas, erodindo a face da berma e depositando na forma de bancos submersos. Ocorre processo inverso entre as tempestades.

A cidade do Recife exibe eventos de erosão costeira, em sua parte sul, com obras de contenção do tipo enrocamento, as quais foram expandidas para direção norte no mês de agosto de 2004. Em estudos realizados por Gregório (2004), considerando perfis morfológicos 
determinados nas praias do Pina e de Boa Viagem, durante os anos de 2002 a 2003, foi possível verificar algumas peculiaridades, por exemplo no perfil próximo à Praça de Boa Viagem, que fica ao norte do enrocamento, percebeu-se um comportamento com grande variação em seu volume sedimentar, com tendências erosivas encontradas no setor $\mathrm{C}$ conforme a Tab 3. Em contrapartida, perfis de praia em equilíbrio, onde existe uma ampla faixa de praia, foram detectados na área central localizada ao norte do setor B.

Tabela 4. Correlação de Pearson, entre os setores B com A, C com B, D com C, em todos os períodos.

\begin{tabular}{|c|c|c|c|c|c|c|c|c|c|c|c|c|c|}
\hline & \multirow{2}{*}{$\begin{array}{l}\text { Setores/ } \\
\text { Períodos }\end{array}$} & \multicolumn{3}{|c|}{$1960-1974$} & \multicolumn{3}{|c|}{$1960-1981$} & \multicolumn{3}{|c|}{$1960-1997$} & \multicolumn{3}{|c|}{$1960-2008$} \\
\hline & & $A$ & $B$ & $\mathrm{C}$ & A & $B$ & C & $A$ & B & $\mathrm{C}$ & A & $\mathrm{B}$ & C \\
\hline \multirow{4}{*}{$\begin{array}{l}\infty \\
\dot{\nu} \\
\stackrel{+}{0} \\
\dot{v}\end{array}$} & $1960-1974$ & $-0,66$ & & & & & & & & & & & \\
\hline & $1960-1981$ & & & & $-0,37$ & & & & & & & & \\
\hline & 1960-1997 & & & & & & & $-0,29$ & & & & & \\
\hline & $1960-2008$ & & & & & & & & & & 0,04 & & \\
\hline \multirow{4}{*}{$\begin{array}{l}u \\
\dot{1} \\
\stackrel{+}{0} \\
\dot{v}\end{array}$} & $1960-1974$ & & $-0,85$ & & & & & & & & & & \\
\hline & $1960-1981$ & & & & & $-0,73$ & & & & & & & \\
\hline & 1960-1997 & & & & & & & & & & & & \\
\hline & $1960-2008$ & & & & & & & & $-0,2$ & & & & \\
\hline \multirow{4}{*}{ 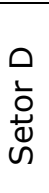 } & $1960-1974$ & & & $-0,32$ & & & & & & & & 0,11 & \\
\hline & $1960-1981$ & & & & & & 0,62 & & & & & & \\
\hline & 1960-1997 & & & & & & & & & $-0,79$ & & & \\
\hline & $1960-2008$ & & & & & & & & & & & & 0,67 \\
\hline
\end{tabular}

Nos setores C e D, a obra de enrocamento, construída durante o ano de 1995, provavelmente tem mantido a linha de costa com um menor recuo, pois neste setor foi encontrado uma diminuição no recuo da linha de costa a partir de 1997. Duarte (2002) realizou um estudo de deslocamento da linha de costa nas praias do Pina e de Boa Viagem, considerando os anos de 1974, 1997 e 1983, como resultado deste estudo no setor A, havia uma tendência geral a progradação.

Pedrosa (2007) estudou a variação de linha de costa da cidade de Olinda, localizada ao norte da cidade do Recife, entre 1915-2004, e verificou que houve um recuo significativo da linha de costa, principalmente entre 1915 e 1970, com o destaque para a parte sul da cidade. Na análise, o referido autor constatou uma área erodida, com um valor aproximado de $95.000 \mathrm{~m}^{2}$, entre 1915 e 2004, nas praias do litoral do município de Olinda. Segundo Muehe and Neves (1995), o processo erosivo deve-se a uma combinação de vários fatores: o déficit de sedimentos fluviais devido à construção de barragens, presença de bancos de arenitos obstruindo o transporte de sedimentos provenientes da plataforma interna e ao longo da costa e obras costeiras construídas em 1915. Porém, Pedrosa (2007) acrescenta outros fatores, como a variabilidade batimétrica bem como a redução das áreas de manguezais entre 1915 e 2004.

As taxas de progradação com maior intensidade encontram-se nos setores B e C durante 1960-1974. No setor A, entre 1960-1974 e 1960-1981 encontraram-se os maiores valores nas 
taxas de retrogradação. Os setores A e C registraram as maiores áreas de recuo, porém no setor C, este recuo foi considerado constante para todo intervalo monitorado. No caso oposto, de acresção da linha de costa os valores encontrados foram relativamente menores em todos os setores quando comparados com as taxas de recuo.

No setor C, a partir de 1960-1974, destaca-se uma contínua perda de sedimentos, que pode estar correlacionado com uma obra de contenção, do tipo enrocamento aderente realizado em 1995 que foi posteriormente expandida para o norte durante a ressaca ocorrida no mês de agosto de 2004. Para o setor $D$, os valores das taxas de retrogradação foram considerados estáveis a partir de 1997, provavelmente devido a construção da obra de contenção.

Os maiores valores de desvio padrão nas médias das taxas de variação da linha de costa foram vistos no setor $A$ (ao norte da área de estudo), e os menores nos setores B e C (ao centro da área de estudo). A maior média das distâncias nos transectos foi a do setor B, entre 19601974 (34,99 m). Os maiores valores negativos nas médias das distâncias dos transectos foram para os casos nos setores A, C e D, principalmente para 1960-1974.

\section{CONCLUSÕES}

O setor A, localizado na praia do Pina, obteve através das análises realizadas retrogradação da linha de costa a partir de 1960-1974, apesar de atualmente existir uma ampla faixa de praia em relação aos setores localizados ao sul da área de estudo, sendo esta taxa de retrogradação menor nos intervalos seguintes.

A parte norte do setor B possui uma larga faixa de praia, se estreitando em direção ao setor C entre 1960-1974, com a progradação da linha de costa nesta época. A partir de 1974 até 2008, foram detectadas pequenas variações da linha de costa, com pouca progradação durante 19601997 e um novo aumento de retrogradação entre 1997 a 2008.

No setor $C$, onde se encontra a obra de contenção do tipo enrocamento, houve um aumento progressivo no recuo da linha de costa considerando 1960-1974 e 1960-1997. Porém, foi percebida uma diminuição na taxa de retrogradação para o ano de 2008. No setor D foram encontrados valores negativos nas taxas de variação da linha de costa e este setor apresentou uma área de recuo contínuo em relação aos demais setores.

As taxas de retrogradação calculadas para a área de estudo dão indicativos que mesmo com a presença dos os bancos de arenitos, que ficam emersos durante a maré baixa nos setores $A, B$ e $C$ não foram fatores que inibiram a instalação de processos erosivos. Outro fator interessante de destaque é que não foi possível encontrar uma correlação linear nas taxas de variação da linha de costa, ou seja, podem existir períodos de retrogradação e outros de progradação no mesmo setor, demonstrando a necessidade do monitoramento costeiro assim como estudos a respeito da hidrodinâmica assim como da vulnerabilidade da linha de costa em relação a erosão costeira influenciados pela urbanização. 


\section{AGRADECIMENTOS}

Os autores agradecem ao Projeto MAI/FINEP (Monitoramento Ambiental Integrado) por fornecer a linha de costa de 2008 e o Departamento de Oceanografia da Universidade Federal de Pernambuco pela disponibilidade das fotografias aéreas de 1960 e ao projeto - APQ- 0486$1.07 / 15$ da FACEPE.

\section{REFERÊNCIAS BIBLIOGRÁFICAS}

Baitelli, R., Almeida, L.E.S.B., Toldo Jr., E.E., Barros, C.E., Martins, L.R. and Nicolodi, J.L. (1999), Retreat of the Rio Grande do Sul coastal zone, Brazil, in Fletcher, C. H. and Matthews, J. V. (Eds.), The Non-steady State of the Inner Shelf and Shoreline: Coastal Change on the Time Scale of Decadesto Millennia in the late Quaternary, University of Hawaii, Honolulu, USA, Nov. 9-12, pp.26-32.

Barbosa, J.A., Souza, E.M., Lima Filho, M.F. and Neumann, V.H. (2003), A estratigrafia da Bacia Paraíba: uma reconsideração, Estudos Geológicos, Vol. 13, pp. 89-108.

Bezerra, M.O., Pinheiro, L. and Morais, J.O. (2003), Shoreline Change of the Mucuripe Harbour Zones (Fortaleza-Ceará, Northeast of Brazil) 1972 - 2003, Journal of Coastal Research, Vol. 50, pp. 1163 - 1167.

Bird, E.C.F. (1996), Beach management, Chichester, John Wiley and Sons Ltda, 281p.

Chaves, M.S. (2005), Dinâmica costeira dos campos petrolíferos Macau/Serra, Litoral, Setentrional do estado do Rio Grande do Norte, Tese de Doutorado, Programa de PósGraduação em Geodinâmica e Geofísica, Centro de Ciências Exatas e da Terra, Universidade Federal do Rio Grande do Norte, 117p.

Delibrias, G. and Laborel, J. (1971), Recent variations of the sea level along the Brazilian coast, Quaternária, Vol. 14, pp. 45-49.

Dominguez, J.M.L., Bittencourt, A.C.S.P., Leão, Z.M.A.N. and Azevedo, A.E.G. (1990), Geologia do quaternário costeiro do Estado de Pernambuco, Revista Brasileira de Geociências, Vol. 20 , pp. $208-215$.

Duarte, R.X. (2002), Caracterização morfo-sedimentológica e evolução de curto e médio prazo das praias do Pina, Boa Viagem e Piedade, Recife, Recife/Jaboatão dos Guararapes - PE, Dissertação de Mestrado, Programa de Pós-Graduação em Oceanografia, Centro de Tecnologia e Geociências, Universidade Federal de Pernambuco, 64p.

Esteves, L.S., Toldo Jr., E.E., Dillenburg, S.R. and Tomazelli, L.J. (2002), Long- and Short-Term Coastal Erosion in Southern Brazil, Journal of Coastal Research, Vol. 36, pp. 273-282.

Ferreira, O., Garcia, T., Matias, A., Taborda, R. and Alveirinho Dias, J. (2006), An integrated method for the determination of set-back lines for coastal erosion hazards on sandy shores, Continental Shelf Research, Vol. 26, pp. 1030-1044. 
Gonçalves, R.M. (2010), Modelagem de tendência a curto-prazo da linha de costa através de dados geodésicos temporais utilizando regressão linear, estimativa robusta e redes neurais artificiais, Tese de Doutorado, Programa de Pós-Graduação em Ciências Geodésicas, Universidade Federal do Paraná, 152p.

Gonçalves, R.M., Coelho, L.D.S., Krueguer, C.P. and Heck, B. (2010), Modelagem preditiva de linha de costa utilizando redes neurais artificiais, Boletim de Ciências Geodésicas, Vol. 16, No. 3, pp. 420-444.

Gonçalves, R. M., Awange, J., Krueger, C. P., Heck, B. and Coelho, L. S. (2012a), A comparison between three short-term shoreline prediction models, Ocean and Coastal Management, Vol. 69, No. 1, pp. 102-110.

Gonçalves, R.M., Awange, J. and Krueger, C.P. (2012b), GNSS-based monitoring and mapping of shoreline position in support of planning and management of Matinhos/PR (Brazil), Journal of Global Positioning Systems, Vol. 11, pp. 156-168.

Gonçalves, R.M., Pacheco, A.D.P., Tanajura, E.L.X. and Silva, L.M. (2013), Urbanização costeira e sombreamento na praia de Boa Viagem, Recife-PE, Brasil, Revista de Geografia Norte Grande, Vol. 54, No. 1, pp. 241-255.

Gregório, M.N. (2004), Sedimentologia e morfologia das praias do Pina e da Boa Viagem, Recife $(P E)$ - Brasil, Dissertação de Mestrado, Programa de Pós-Graduação em Oceanografia, Centro de Tecnologia e Geociências, Universidade Federal de Pernambuco, 98p.

Gregório, M.N., Araújo, T.C.M. and Valença, L.M.M. (2004), Variação sedimentar das praias do Pina e Boa Viagem, Recife (PE) - Brasil, Tropical Oceanography, Vol. 32, No. 1, pp. 39-52. Gregório, M.N. and Araújo, T.C.M. (2008), Interannual morphological changes of Boa Viagem beach- Northeast coast of Brazil, Boletim Paranaense de Geociências, Vol. 62-63, pp. 6184.

Grigio, A.M. (2003), Aplicação de sensoriamento remoto e sistema de informação geográfica na determinação da vulnerabilidade natural e ambiental do município de Guamaré (RN): simulação de risco às atividades da indústria petrolífera, Dissertação de Mestrado, PósGraduação em Geodinâmica e Geofísica, Centro de Ciências Exatas e da Terra, Universidade Federal do Rio Grande do Norte, 233p.

Instituto Brasileiro de Geografia e Estatística (IBGE) (1997), Geografia do Brasil, Região Nordeste, em: http://www.ibge.gov.br/home/geociencias/geografia/default.shtm (acesso em 18 de Setembro de 2015).

Krueger, C.P., Gonçalves, R.M. and Heck, B. (2009), Surveys at the Coast of Paraná, Brazil, to Determinate the Temporal Coastal Changes, Journal of Coastal Research, Special Issue 56, pp. 632-635.

Krueger, C.P., Soares, C.R., Huinca, S.M., Leandro, D. and Gonçalves, R.M. (2011), Satellite positioning on the coast of the Parana, Brazil, Journal of Coastal Research, Special Issue 64, pp. $1352-1356$. 
Lima, Z.M.C., Alves, A.L., Amaro, V.E. and Vital, H. (2001), Evolução da linha de costa do esporão de Galinhos-RN usando fotografias aéreas e imagem Landsat, Pesquisas em Geociências, Vol. 28, No. 2, pp. 497-507.

Lima, Z.M.C. (2004), Caracterização da dinâmica ambiental da região costeira do município de Galinhos, litoral setentrional do Rio Grande do Norte, Tese de Doutorado, Pós-Graduação em Geodinâmica e Geofísica, Centro de Ciências Exatas e da Terra, Universidade Federal do Rio Grande do Norte, 144p.

Mabesoone, J.M. (1964), Origin and age of the sandstone reefs of Pernambuco (Northeastern Brazil), Journal of Sedimentary Petrology, Vol. 34, pp. 715-726.

Martin, L., Dominguez, J.M.L. and Bittencourt, A.C.S. (1998), Climatic control of coastal erosion during a sea-level fall episode, Anais da Academia Brasileira de Ciências, Vol. 70, No. 2, pp. 249-266.

Mazzer, A.M. and Dillenburg, S. (2009), Variações temporais da linha de costa em praias arenosas dominadas por ondas do sudeste da Ilha de Santa Catarina (Florianópolis, SC, Brasil), Pesquisas em Geociências, Vol. 36, No. 1, pp. 117-135.

Mendonça, F.J.B. (2005), Posicionamento de alta resolução - adequação e aplicação à morfologia costeira, Tese de Doutorado, Programa de Pós-Graduação em Oceanografia, Centro de Tecnologia e Geociências, Universidade Federal de Pernambuco, 94p.

Mendonça, F.J.B., Gonçalves, R.M., Awange, J., Silva, L.M. and Gregório, M.N. (2014), Temporal shoreline series analysis using GNSS, Boletim de Ciências Geodésicas, Vol. 20, No. 3, pp. 711-729.

Miller, J.K. and Dean, R.G. (2004), A simple new shoreline change model, Coastal Engineering, Vol. 51, No. 7, pp. 531-556.

Muehe, D. and Neves, C.F. (1995), Potential impact of sea-level rise on the metropolitan region of Recife, Brazil, Journal of Coastal Research, Special Issue 14, pp. 116-13.

Muehe, D. (1998), Geomorfologia costeira, in Guerra, A.J.T. and Cunha, S.B. (Eds.), Geomorfologia do Brasil, Rio de Janeiro, Bertrand Brasil, pp. 253-306.

Oliveira, S.C. (2005), Evolução recente da linha de costa no troço costeiro Forte Novo - Garrão (Algarve), Dissertação de Mestrado, Faculdade de Ciências, Universidade de Lisboa, 115p.

Pedrosa, F.A. (2007), Aspectos da evolução da linha de costa e da paisagem litorânea do Município de Olinda entre 1915 e 2004: evidências do tecnógeno em Pernambuco, Tese de Doutorado, Programa de Pós-Graduação em Geociências, Centro de Tecnologia e Geociências, Universidade Federal de Pernambuco, 175p.

Ribeiro, G.P., Rocha C.H.O., Figueiredo Jr., A.G., Silva, C.G., Silva, S.H.F., Moreira, P.S.C, Guimarães, M.S.D., Pereira, A.P., Almeida, A.G., Pinna, B.G., Souza, C.F., Silva, C., Santos, R.A. and Vasconcelos, S.C. (2004), Análise espaço-temporal no suporte à avaliação do processo de erosão costeira em Atafona, São João da Barra (RJ), Revista Brasileira de Cartografia, Vol. 56, No. 2, pp. 129- 138. 
Rollnic, M. (2002), Hidrologia, clima de onda e transporte advectivo na zona costeira de Boa Viagem, Piedade, Candeias - PE, Dissertação de Mestrado, Programa de Pós-Graduação em Oceanografia, Centro de Tecnologia e Geociências, Universidade Federal de Pernambuco, $111 \mathrm{p}$.

Santos, U.S.T. (2008), Avaliação geoambiental das praias do Município dos Guararapes, região metropolitana do Recife, Dissertação de Mestrado, Programa de Pós-Graduação em Oceanografia, Centro de Tecnologia, Universidade Federal de Pernambuco, 111p.

Schwarzer, K. and Schrottke, K. (1999), Shoreline displacement along the Southern Baltic Sea coastline due to sea-level changes and human impact, in Fletcher, C.H. and Matthews, J.V. (Eds.), The Non-steady State of the Inner Shelf and Shoreline: Coastal Change on the Time Scale of Decadesto Millennia in the late Quaternary, University of Hawaii, Honolulu, USA, Nov. 9-12, pp. 150-152.

Silva, A.J.S., Barbosa, S.C.T., Leal, M.V., Lins, A.R. and Costa, M.F. (2006), Ocupação da praia da Boa Viagem Recife/PE) ao longo de dois dias de verão: um estudo preliminar, PanAmerican Journal of Aquatic Sciences, Vol. 1, No. 2, pp. 91- 98.

Silva, L.M., Gonçalves, R.M., Lira, M.M.S. and Pereira, P.S. (2013), Modelagem fuzzy aplicada na detecção da vulnerabilidade à erosão costeira, Boletim de Ciências Geodésicas, Vol. 19, pp. 746-764.

Sousa, C.M.F. (2004), A integração do sistema GPS/INS para a monitorização da linha de costa do litoral do Algarve, Dissertação de Mestrado, Faculdade de Ciências, Universidade de Lisboa, 84p.

Tanajura, E.L.X., Gonçalves, R.M. and Krueger, C.P. (2011), Análise da acurácia dos métodos cinemáticos de posicionamento GPS em aplicações costeiras, Boletim de Ciências Geodésicas, Vol. 17, pp. 23-36.

Tessler, M.G. and Goya, S.C. (2005), Processos costeiros condicionantes do litoral brasileiro, Revista do Departamento de Geografia, Vol. 17, pp. 11-23.

Thieler, E.R., Himmelstoss, E.A., Zichichi, J.L. and Ergul, A. (2009), Digital Shoreline Analysis System (DSAS) version 4.0 - An ArcGIS extension for calculating shoreline change*. U.S. Geological Survey: Open-File Report, Series Number 2008-1278. *current version 4.3, 2009.

Toldo Jr. E.E., Almeida, L.E.S.B., Nicolodi J.L. and Martins, L.R. (2005), Retração e progradação da zona costeira do estado do Rio Grande do Sul, Gravel, Vol. 3, pp. 33-38.

Villwock, J.A., Lessa, G.C., Suguio, K., Angulo, R.J. and Dillenburg, S.R. (2005), Geologia e geomorfologia de regiões costeiras, In Souza, C.R.G., Suguio, K., Oliveira, A.M.S. and Oliveira, P.E. (Eds.), Quaternário do Brasil, Ribeirão Preto, Holos, pp. 52-74. 